\title{
Medial Hemisoleus Muscle Flap in Reconstruction of Soft Tissue Defects of Lower Leg
}

Mohamed Makboul, Ahmed Mousa, Mohamed Elotiefy and Mohamed Hamdy Mohamed Ahmed

Plastic Surgery Department, Faculty of Medicine - Aswan University

Corresponding author: Mohamed Hamdy Mohamed Ahmed; Mobile: (+20)01099050142, Email: o.hamdey@ gmail.com

\begin{abstract}
Background: Reconstruction of lower leg represents a challenge task for plastic surgeons. Although microsurgical flaps have been the golden choice for this reconstruction, many hospitals do not have equipment or microsurgical staff trained for this type of procedure.

Aim: The objective of the current study is to evaluate of clinic applicability and functional outcome results of medial hemisoleus muscle as a reconstructive flap modality in lower third leg soft tissue defect. This includes clinical study comparison between two surgical modalities of hemisoleus muscle Hap elevation.

Methodology: This study was conducted in Plastic Surgery Department, Aswan University Hospital from May 2017 to August 2018. It was a descriptive study including 30 patients (23 males and 7 females with the age ranging between 29 and 51 years). Results: Our study confirmed this fact as preservation of lateral portion is suitable for planter flexion with gastrocnemius muscle and medial hemisoleus muscle flap showing long arc of rotation especially distally based posterior tibial artery medial hemisoleus muscle flap has long arc of rotation cover up to heal defect. Conclusion: Medial hemisoleus muscle flap is a reliable option for the reconstruction of soft tissue defects of lower leg based on minor pedicle or reversed flow based on posterior tibial artery. Careful flap dissection with preservation of as many perforators as possible is the key to success.
\end{abstract}

Keywords: Lower leg, soft tissue defects, medial hemisoleus muscle flap, reversed flow medial hemisoleus muscle flap, distally based perforator medial hemisoleus muscle flap.

\section{INTRODUCTION}

Reconstruction of the lower leg represents a challenge for many plastic surgeons. Although the microsurgical flap has been the method of choice for this reconstruction ${ }^{(1-4)}$, many hospitals do not have equipment or microsurgical staff.

In other cases, the patients are not candidates for microsurgical reconstruction because of their medical conditions, and the surgeon is therefore required to use alternative methods of reconstruction. Often, defects in the lower leg quickly result in the exposure of relevant structures such as bone and tendon, the coverage of which requires the performance of a safe reconstruction method with a well-vascularized tissue $^{(2,3)}$.

The soleus muscle is located in the posterior compartment of the leg anterior to the gastrocnemius muscles, and is classified as type II muscle according to the classification of Mathes and Nahai. Medial head of soleus dominant pedicle arises from posterior tibial artery and peroneal artery and minor pedicle arises from posterior tibial artery ${ }^{(2,3)}$.

The soleus muscle is frequently used for the reconstruction of defects of the middle third of the leg, based on its major pedicle. However, the viable use of the soleus muscle in a reverse manner based on posterior tibial artery (dominant and minor pedicles) or distally dased manner on its minor pedicle only, has been described by several authors for the reconstruction of defects of the lower third of the leg as an alternative to the use of microsurgical flaps ${ }^{(1-5)}$.

An advantage of using the hemisoleus flap rather than the whole soleus muscle flap is the preservation of plantar flexion of the foot performed by the lateral portion of the muscle, which is left in its original location. Moreover, the medial flap has a greater arc of rotation than that of a conventional soleus muscle flap ${ }^{(\mathbf{1 - 3})}$. The medial part of the muscle is supplied in its whole length by perforators of the posterior tibial artery. Because of this constant arterial supply, the medial part of the soleus muscle is viable as a muscle flap distally based or a reversed flow manner ${ }^{(1-3)}$. This study reports thirty cases of reconstruction of defects of the lower leg with the use of the medial hemisoleus muscle flap.

\section{THE AIM OF THE STUDY}

The objective of the current study is to evaluate of clinic applicability and functional outcome results of medial hemisoleus muscle as a reconstructive flap modality in lower third leg soft tissue defect. This includes clinical study comparison between two surgical modalities of hemisoleus muscle Hap elevation.

\section{PATIENTS AND METHODS}


This study was conducted in Plastic Surgery Department, Aswan University Hospital from May 2017 to August 2018.

The study was approved by the Ethics Board of Al-Azhar University.

It was a descriptive study including 30 patients (23 males and 7 females with the age ranging between 29 and 51 years). All these patients were presented to trauma unit or outpatient clinic of plastic surgery. Defect size was variable (Table-1, see the results). Soft tissue coverage was provided with medial hemisoleus muscle flap, all patients were divided to two groups; each group of 15 patients:

Group I: defect reconstructed with distally based perforator medial hemisoleus muscle flap.

Group II: defect reconstructed with reversed posterior tibial artery hemisoleus muscle flap.

All the patients were followed up for a minimum of six weeks.

\section{Surgical Technique}

All the patients were operated upon in supine position with external rotation and abduction at hip joint and slight flexion at knee joint. Tourniquet was applied in all the patients.

The soleus muscle can be approached for medial exposure through a medial incision $2 \mathrm{~cm}$ posterior to the medial edge of the tibia, some cases dissection occur through an existing wound along the medial border of the tibia. Dissection proceeds through the skin and subcutaneous layer cut with haemostasis, then the soleus muscle is identified (Figure 1A), both the gastrocnemius and plantaris tendons lie superficial to the soleus muscle. The medial origins of the muscle at medial tibial border is dissected gently (Figure 1B), exposing the flexor digitorum longus, posterior tibial artery and nerve, and the flexor halluces longus muscles. Superficially, the soleus muscle is separated from the overlying gastrocnemius and skin, and subcutaneously it is separated more distally to the surface of the Achilles tendon, then the muscle is retracted outward.

\section{For group I:}

Careful identification of minor pedicle is important (Figure 1C) to select most suitable sizable perforators and selection of most dominant perforator upon it flap can be supplied. The site of muscle division by cautery would depend on the size of defect and degree of flap rotation. Dimensions of the defect were measured to determine the level of the muscle proximally at which it can be divided. The medial half of the soleus muscle was splitted longitudinally from lateral half at the level of midline raphe. The median raphe was located and divided, and the flap was dissected from proximal to distal, ligating segmental vessels as needed for rotation of the flap (Figure 1C and 2).

\section{For group II:}

After complete separation of soleus muscle from its origin dissection and identification of the soleus muscle, careful and complete dissection of posterior tibial artery along its course within the muscle with preservation of muscle perforators of dominant and minor pedicles of the muscle to preserve muscle blood supply was done, then muscle raphe was separated completely, then ligation of posterior tibial artery could be done (Figure 3).

So the muscle with posterior tibial artery became completely free, blood flow to the muscle depending on back flow of posterior tibial artery through dominant and minor pedicles of the muscle.

Flap was transferred to the defect and secured to the edges of the defect with Vicryl sutures. Donor site was closed over a suction drain. A split thickness skin graft was taken by the knife from the anteromedial aspect of the thigh of the same operating limb or from the contralateral thigh, meshing of skin graft was done to avoid skin graft loss due to hematoma formation under graft, and graft fixation with skin stables was done. Above knee slab was applied for one week in every patient to avoid skin graft loss due to underlying muscle movements. Post operatively patients were advised to elevate their operated limbs to reduce pain and swelling. Good dressing of donor site of muscle flap and recipient site of flap was done with Vaseline gauze and bulky dressing was applied over it then rolled with gauze and creep bandage, the donor site of skin graft dressing was done by Vaseline gauze and bulky dressing was done carefully with complete aseptic technique. Careful transportation of the patient was done with adequate positioning of the operated limb to avoid compression of the pedicle of the flap.
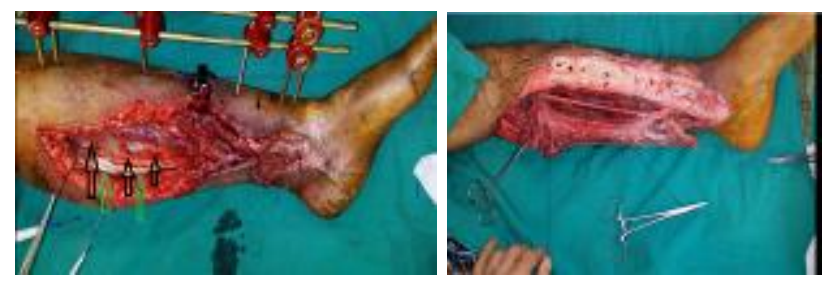
Medial Hemisoleus Muscle Flap...

A

B

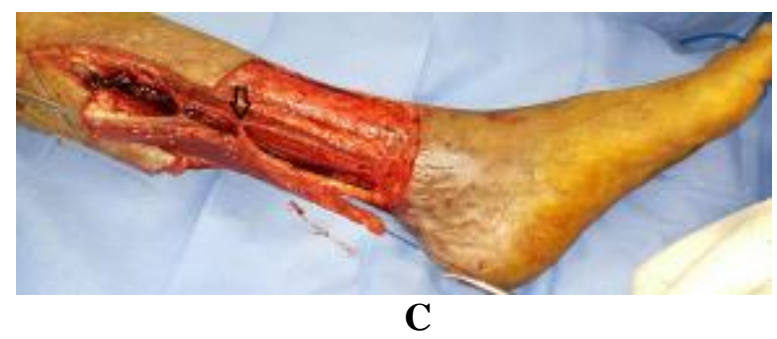

Figure 1 - Surgical technique. In A, Identification of soleus (Green arrow. Gastrocnemius; Black arrow. Soleus muscle). In $\mathbf{B}$, Soleus muscle separation from tibial origin. In $\mathbf{C}$, Soleus muscle Minor pedicle identification.

\section{Statistical analysis}

Recorded data were analyzed using the statistical package for social sciences, version 20.0 (SPSS Inc., Chicago, Illinois, USA). Quantitative data were expressed as mean \pm standard deviation (SD). Qualitative data were expressed as frequency and percentage.

\section{The following tests were done:}

When comparing between two means: Independent-samples t-test of significance was used.

Chi-square $\left(\mathrm{x}^{2}\right)$ test of significance was used in order to compare proportions between qualitative parameters.

The dependability interval was set to $95 \%$ and the margin of error accepted was set to $5 \%$. So, the $p$-value considered significant as the following:

- P-value <0.05 was considered significant.

- P-value $<0.001$ was considered as highly significant.

- P-value $>0.05$ was considered insignificant.

\section{RESULTS}

In our study we operated upon 30 patient presented with lower leg defect; 19 patient post motorcar accident, 8 patients exposed tibial plate post tibial fixation, 3 patient post Marjolin's ulcer excision. Our study revealed no statistically significant differences in demographic between the two groups. Site of skin defect; 14 patients with skin defect of lower third of the leg (46.7\%), 12 patients with skin defect around ankle joint (40.0\%), 4 patients with skin defect Lower third of the leg+ around ankle joint (13.3\%). Mean age of patients was 37.32 years.

Flap viability assessment showed; (Table 1) 23 patients showing viable medial hemisoleus muscle flap (76.7\%), 5 patients showing mild congested flap which improved spontaneously (16.7\%), and 2 patients $(6.7 \%)$ showing loss of about $2 \mathrm{~cm}$ distal part of (tip) flap. But both techniques showed no flap loss, congested flaps showed subside of congestion and flaps became viable.
So both techniques are versatile for medial hemisoleus muscle flap design. The choice between the two techniques depended on site and size of defect.

About 19 patients $(63.3 \%)$ showed complete graft taken, 6 patients $(20 \%)$ showed partial loss of skin graft, patients management done conservatively that healed completely within 10 days. Five patients $(16.7 \%)$ showed complete skin graft loss that necessitated another skin grafting session. Lower limb assessment showed no functional motor deficit after hemisoleus muscle harvesting (Figure 4).

Advantages and disadvantages of both techniques: Advantages of distally based perforator medial hemisoleus muscle flap:

1. Long arc of rotation.

2. Restoration of planter flexion of foot.

3. Bulky flap suitable for obliteration of dead space.

4. Good blood supply so compete osteomyelitis.

5. No scarification of major blood vessels.

Disadvantages of distally based perforator medial hemisoleus muscle flap:

1. Limited option for lower leg only due to relatively short arc of rotation.

2. Needs careful dissection an identification of dependable perforator.

Advantages of reversed flow posterior tibial artery medial hemisoleus muscle flap:

1. Long arc of rotation suitable option for defects around ankle joint.

2. Restoration of planter flexion of foot.

3. Bulky flap suitable for obliteration of dead space.

4. Good blood supply so compete osteomyelitis.

Disadvantages of reversed flow posterior tibial artery medial hemisoleus muscle flap:

1. Scarification of major blood vessels (posterior tibial artery). 
Mohamed Makboul et al.

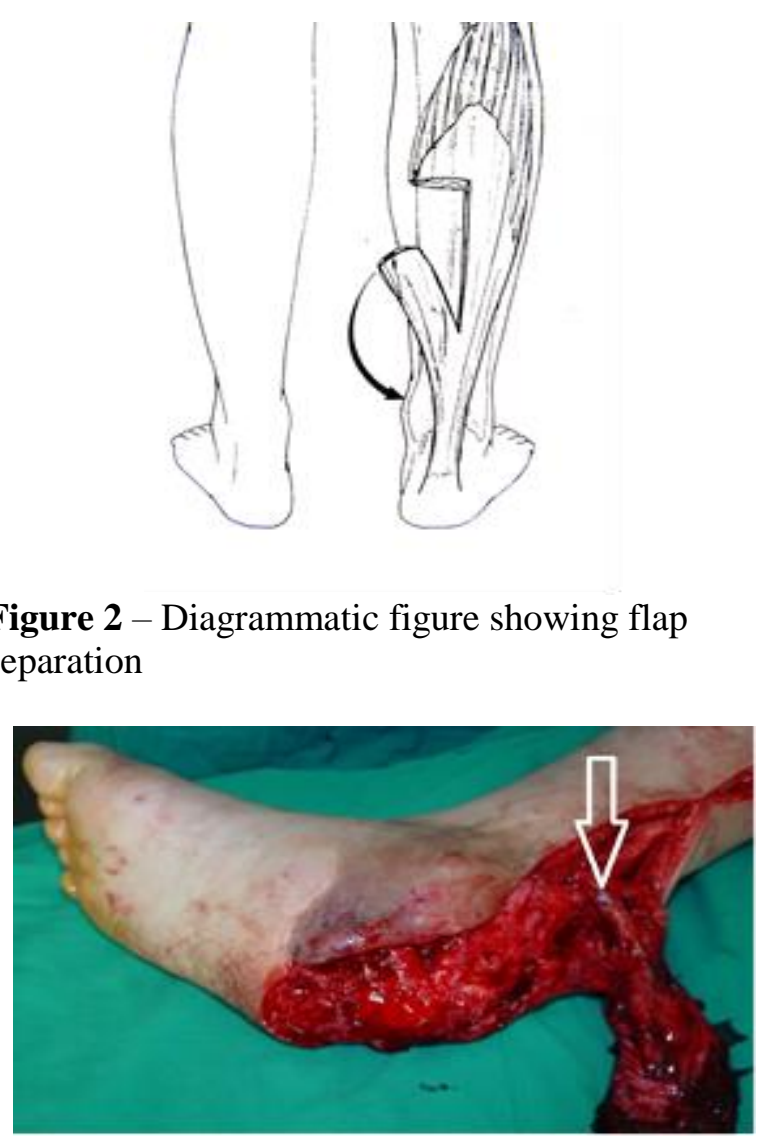

dissection within posterior tibial artery (Arrow directed to posterior tibial artery).

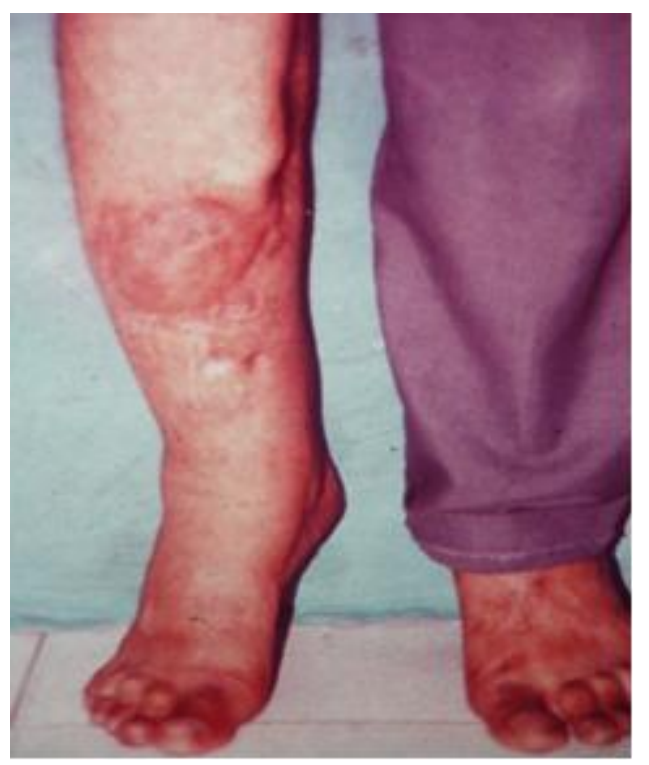

Figure (4): photo showing ability of patient to stand up over his foot indicating no functional defici

Figure 2 - Diagrammatic figure showing flap separation

Figure (3): Medial hemisoleus muscle flap after

Table 1: Flap Viability and Graft taken between 2 study groups

\begin{tabular}{|c|c|c|c|c|c|c|c|}
\hline & \multicolumn{4}{|c|}{ Groups } & \multirow{2}{*}{\multicolumn{2}{|c|}{ sher's Exact test of significance }} \\
\hline & & \multicolumn{2}{|c|}{ Group 1} & \multicolumn{2}{|c|}{ Group 2} & & \\
\hline & & $\mathrm{N}$ & $\%$ & $\mathrm{~N}$ & $\%$ & $\mathrm{P}$-value & Sig. \\
\hline \multirow{3}{*}{$\begin{array}{l}\text { Flap } \\
\text { iability }\end{array}$} & Viable & 12 & $80.0 \%$ & 11 & $73.3 \%$ & \multirow{3}{*}{1.00} & \multirow{3}{*}{ NS } \\
\hline & Congested & 2 & $13.3 \%$ & 3 & $20.0 \%$ & & \\
\hline & pss of distal part of flap & 1 & $6.7 \%$ & 1 & $6.7 \%$ & & \\
\hline \multirow{3}{*}{ aft taken } & Taken & 10 & $66.7 \%$ & 9 & $60.0 \%$ & \multirow{3}{*}{0.762} & \multirow{3}{*}{ NS } \\
\hline & partially lost & 2 & $13.3 \%$ & 4 & $26.7 \%$ & & \\
\hline & completely lost & 3 & $20.0 \%$ & 2 & $13.3 \%$ & & \\
\hline
\end{tabular}

\section{Cases examples}

\section{CASE 1 (Figure 5)}

A 29-year-old male patient had exposed tibial plate in the distal third of the right leg that had not healed for one year following a car accident. During the physical examination, a lesion of $3.5 \mathrm{~cm} \times 4 \mathrm{~cm}\left(14 \mathrm{~cm}^{2}\right)$ was seen. On August 15, 2017, the patient underwent wide debridement of the lesion, including the exposed bone portion, in which reconstruction of the defect was performed with the distally based perforator medial hemisoleus muscle flap according to the described technique. The patient was discharged on the second postoperative day. The flap showed total survival and was grafted on the seventh postoperative day, resulting in total integration of the skin graft. The lesion has not recurred up to the present date. 


\section{CASE 2 (Figure 6)}

A 38-year-old male patient had a lesion in the distal third of the right leg following a car accident over the heel, skin graft was done at March 13, 2018 patient presented with heel ulcer. During the physical examination, a lesion of $2.5 \mathrm{~cm} \times 2 \mathrm{~cm}\left(5 \mathrm{~cm}^{2}\right)$ was identified with bone exposure in the central portion. Arteriography of the right lower limb, performed before the reconstruction, revealed absence of distal vascular impairment, and the patient underwent reconstruction of the defect with the reversed flow medial hemisoleus muscle flap.

The hemisoleus flap production was suitable for coverage of the bone defect. The patient was discharged on the second postoperative day. The flap showed total survival and resulting in total integration of the skin graft.

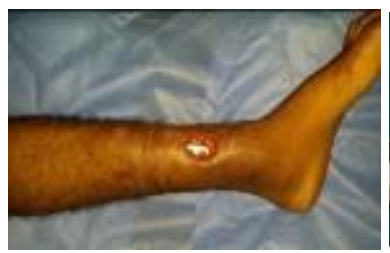

A

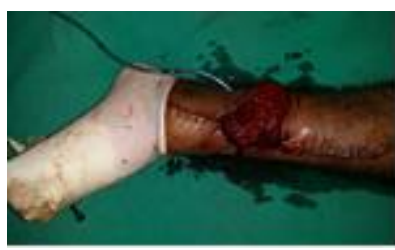

B

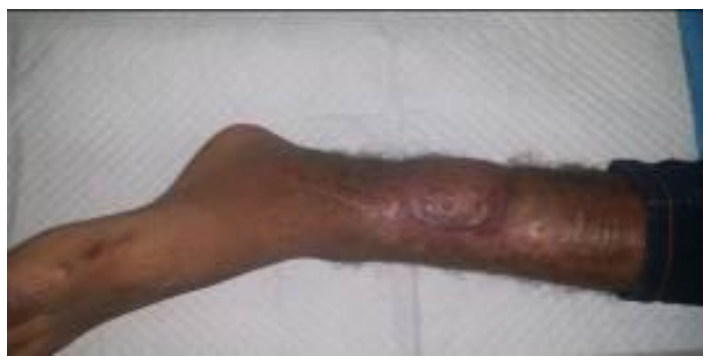

$\mathrm{C}$

Figure (5): Case showing exposed tibial plate lower leg covered with distally based perforator medial hemisoleus muscle flap. In A showing exposed tibial plate of lower third leg post tibial fracture fixation. In B showing reconstruction of lower third defect with hemisoleus muscle flap, intraoperative photo. In $\mathbf{C}$ showing partial skin graft loss.

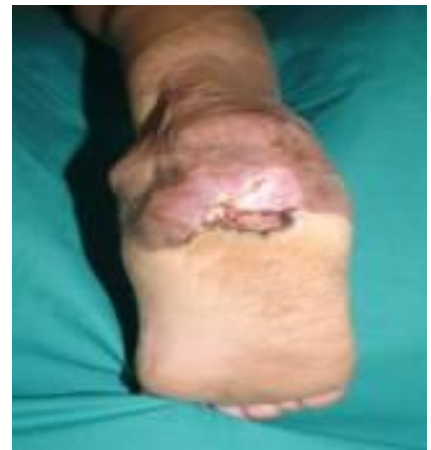

A

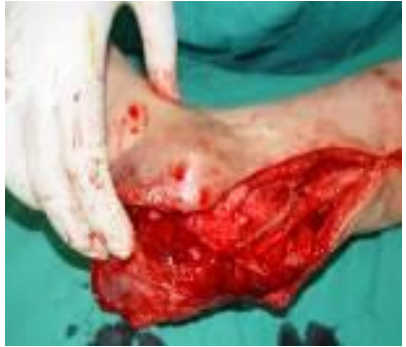

C

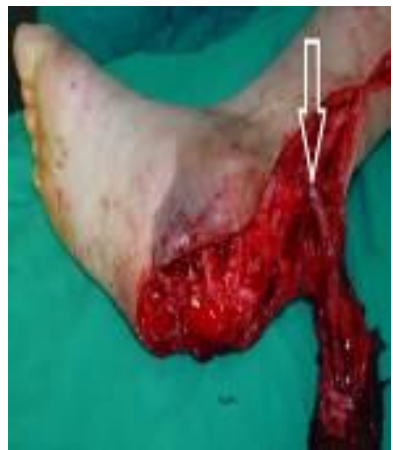

$\mathbf{B}$

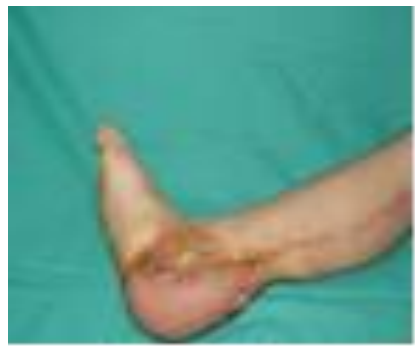

D

Figure (6): A case of heal ulcer covered with reversed flow medial hemisoleus muscle flap; In A preoperative photo.

In $\mathbf{B}$ intraoperative photo after flap elevation arrow directed to posterior tibial artery. In $\mathbf{C}$ After flap positioning to the defect. In $\mathbf{D}$ postoperatively showing graft taken 


\section{DISCUSSION}

The bipennate nature of the soleus muscle and the independent neurovascular supply to both medial and lateral halves of the muscle are the key anatomical factors that allow splitting of the muscle longitudinally along its raphe ${ }^{(6)}$. These anatomical features allow longitudinal muscle splitting without compromising its blood supply. On the same time a beg benefit could be gained by preservation good planter ankle flexion.

In 2011, De Souza et al. ${ }^{(7)}$ reported that medial hemisoleus muscle flap was selected as an alternative to whole soleus. Medial hemisoleus muscle flap was given preference over the whole soleus muscle because of its longer arc of rotation and minimal functional loss of foot plantar flexion than using the whole muscle. Our study confirmed this fact as preservation of lateral portion is suitable for planter flexion with gastrocnemius muscle and medial hemisoleus muscle flap showing long arc of rotation especially distally based posterior tibial artery medial hemisoleus muscle flap has long arc of rotation cover up to heal defect.

As medial hemisoleus muscle flap is less bulky than the whole soleus muscle flap, the reconstructive outcome was usually cosmetically better than using the whole muscle ${ }^{(6)}$. Rotated hemisoleus muscle showed atrophy within few months. This shrinkage added to give reconstructed area more pleasing outcome.

The most significant advantage of hemisoleus muscle flap was preservation of foot plantar flexion power by the hemisoleus muscle belly left in situ. The medial half of the muscle is supplied constantly throughout its length by the perforators from the posterior tibial artery ${ }^{(6)}$. We agree with this fact as soleus muscle is the powerful planter flexors and one half of the muscle is enough to maintain of planter flexion of foot.

Distally based hemisoleus muscle flap is a good option but it carried a relatively flap complication incidence. This was explained by variation in the number and site of its distal perforators. Several anatomical studies were carried on the vascular anatomy of the soleus muscle minor pedicles. The number of perforators was extremely variable. However, no available reports talked about total absence of distal muscle perforators ${ }^{(8)}$. Proper understanding of the patterns of blood supply of soleus muscle flap not only made flap survival high, but also allowed variable useful flap designs. This will be useful for lower leg defects.
The reverse hemisoleus muscle flap, initially proposed by De Souza ${ }^{(7)}$, involved the use of the medial part of the soleus muscle for reconstruction of the medial and distal parts of the lower limb with narrow tibial exposures and irrigation based on the posterior tibial artery perforators but did not gain popularity due to harvesting of major vessel and at high total ischemia rate ${ }^{(7)}$. On our study proper perforator identification for soleus muscle enabled us to design flap safely in various manners specially reversed flow posterior tibial artery medial hemisoleus muscle flap and this was the key to ensure flap viability and versatility.

In 2003, Beck et al. ${ }^{(5)}$ compared several flaps for treatment of defects of the leg by using the gastrocnemius muscle, fasciocutaneous flaps, free flaps, and reverse soleus flap. The reverse medial hemisoleus muscle flap was the only flap in this series that did not present complications, and the authors concluded that it is an excellent option when its use is possible ${ }^{(5)}$. This also was proved as reversed flow hemisoleus showed long arc of rotation, no functional deficit of planter flexion and good blood supply with less possibility of ischemia. Also this well-nourished flap provided good blood flow to wound defect with antibiotic deposition minimizing wound and bone healing problems and overcome of osteomyelitis ${ }^{(7)}$.

There are reports that showed that distally based flap can survive on only one or two perforators $^{(7)}$. That is the basic principle of distally perforator muscle flap and explained by angiosome theory of blood supply to distal muscle portion.

In cases of fasciocutaneous flaps, in which the reverse sural artery flap is used, the major limitation is venous congestion. This leads to problems in flap viability associated with compression of the pedicle when a subdermal tunnel is used for transposition and positioning. In comparison the reverse medial hemisoleus muscle flap venous congestion is less than fasciocutaenous flap as venous return is through muscle pump and venae committantaes ${ }^{(7)}$. Medial hemisoleus muscle flap showed mild to moderate congestion in few cases, which mostly resolved without flap necrosis.

In 1995, Yajima et al. ${ }^{(9)}$ reported that the most distal soleus perforator was found at fixed distance of about $61-145 \mathrm{~mm}$ above the medial malleolus. To increase the number of perforators and to augment the vascular supply of the distally based hemisoleus flap, the reversed flow hemisoleus flap was described. 
In 1982, by Guyuron et al. ${ }^{(10)}$ however, the flap did not gain much popularity because it has the great disadvantage of sacrificing a major leg blood vessel and cannot also be used if there is associated vascular injury or disease in the same leg. This difference between two flaps variant seems to be true in the past, but now after great understanding of anatomy of soleus muscle result of both flap variant showing the same result ${ }^{(\mathbf{8})}$.

In addition to the advantages already discussed, another relevant point is the hospital stay and cost benefit of the procedure. In 2005, Thornton et al. ${ }^{(11)}$ compared the hospital costs between the reverse soleus flap and microsurgical flaps in patients with similar profiles and defects and observed that the hospitalization time, surgical time, hospital expenses, and the use of the intensive care unit were significantly lower in the group of patients undergoing soleus flap reconstruction if compared with free flap procedure ${ }^{(7)}$. This result according to available options of reconstruction and hospital facilities and economic state and patient general condition but in general cost, surgical time and procedures of medial hemisoleus muscle flap was also easier than microsurgical techniques.

\section{CONCLUSIONS}

Medial hemisoleus muscle flap is a reliable option for the reconstruction of soft tissue defects of lower leg based on minor pedicle or reversed flow based on posterior tibial artery.

Careful flap dissection with preservation of as many perforators as possible is the key to success.

It has longer arc of rotation, easier to inset for a variety of lower leg defects compared to the whole soleus and less bulky, so provides a better contour of reconstruction. Distally based hemisoleus flap has upper advantage over reversed flow hemisolus muscle flap due to preservation of posterior tibial artery, although both techniques showed excellent result in coverage such defects in lower leg defects.

\section{REFERENCES}

1. Pu LL (2006): The reversed medial hemisoleus muscle flap and its role in re-construction of an open tibial wound in the lower third of the leg. Ann Plast Surg., 56(1):59-63.

2. Pu LL (2007): Soft-tissue reconstruction of an open tibial wound in the distal third of the leg: a new treatment algorithm. Ann Plast Surg., 58(1): 78-83.

3. Pu LL (2005): Successful soft-tissue coverage of a tibial wound in the distal third of the leg with a medial hemisoleus muscle flap. Plast Reconstr Surg., 115(1):245-51.

4. Pu LL (2008): Further experience with the medial hemisoleus muscle flap for soft-tissue coverage of a tibial wound in the distal third of the leg. Plast Reconstr Surg., 121(6):2024-8.

5. Beck JB, Stile $F$ and Lineaweaver W (2003): Reconsidering the soleus muscle flap for coverage of wounds of the distal third of the leg. Ann Plast Surg., 50(6):631-5

6. Tarar MN, Malik FS, Khalid $\mathrm{K}$ et al. (2009): Hemisoleus muscle flap, a better option for coverage of open fractures involving middle third of tibia. Journal of Ayub Medical College Abbottabad, 21(4): 154-158.

7. De Souza Filho MV, De Oliveira Teixeira JC and De Castro OC (2011): Reversed hemisoleus flap for wound coverage in the distal third of the leg: retalho hemissolear reverso na reconstrução de defeito do terço distal da perna. Revista Brasileira de Cirurgia Plástica., 26(4): 710-713.

8. Zakaria Khaled Sief EL-Din (2010): The Use of Island Soleus Muscle Flap in Reconstruction of Pretibial Skin Defects. Egypt, J Plast Reconstr Surg., 34(1): 37-40.

9. Yajima $H$, Tamai $S$, Ishida $H$ et al. (1995): Partial soleus muscle island flap transfer using minor pedicles from the posterior tibial vessels. Plastic and Reconstructive Surgery, 96(5): 1162-1168.

10. Guyuron B, Dinner MI, Dowden RV et al. (1982): Muscle flaps and the vascular detour principle: the soleus. Annals of Plastic Surgery, 8(2): 132-140.

11. Thornton BP, Rosenblum WJ and Pu LL (2005): Reconstruction of limited soft-tissue defect with open tibial fracture in the distal third of the leg: a cost and outcome study. Annals of Plastic Surgery, 54(3): 276280. 